\title{
Primary prevention of anxiety disorders in primary care: A systematic review
}

\author{
Javier García-Campayo ${ }^{\mathrm{a}, *}$, Yolanda López del Hoyo ${ }^{\mathrm{b}}$, Montserrat Salas Valero ${ }^{\mathrm{c}}, \mathrm{M}^{\mathrm{a}}$ Cruz Pérez Yus ${ }^{\mathrm{d}}$, \\ Eva Andrés Esteban ${ }^{\text {, }}$, Marta Puebla Guedea ${ }^{f}$, Rosa Magallón Botaya ${ }^{\mathrm{g}}$
}

a Department of Psychiatry, Miguel Servet University Hospital, University of Zaragoza, REDIAPP, Isabel la Catolica, 1-3, 50009 Zaragoza, Spain

${ }^{\mathrm{b}}$ Department of Psychology \& Sociology, University of Zaragoza, REDIAPP, Violante de Hungría, 23, 50009 Zaragoza, Spain

${ }^{c}$ Aragon Health Sciences Institute, San Juan Bosco, 13, 50009 Zaragoza, Spain

d Aragon Health Sciences Institute, REDIAPP, CS. Arrabal, Andador Aragues Puerto, 2-4, 50015 Zaragoza, Spain

e 12 de Octubre Research Institute, Avda. de Córdoba, s/n, 28041 Madrid, Spain

${ }^{\mathrm{f}}$ REDIAPP, CS, Arrabal, Andador Aragues Puerto, 2-4, 50015 Zaragoza, Spain

${ }^{g}$ Health Aragon, REDIAPP, CS, Arrabal, Andador Aragues Puerto, 2-4, 50015 Zaragoza, Spain

\section{A R T I C L E I N F O}

Available online 16 October 2014

\section{Keywords:}

Anxiety disorders

Primary prevention

Primary health care

\begin{abstract}
A B S T R A C T
Objective. Anxiety disorders are the most prevalent psychiatric disorders in primary care and have significant social, economic, and interpersonal costs. Primary care is an ideal setting to prevent the appearance of anxiety disorders. The aim of this study was to evaluate the efficacy and cost-effectiveness of primary prevention interventions in anxiety disorders in the adult population receiving primary health care.

Methods. A literature search was carried out in four databases-PubMed, PsycInfo, Cochrane, and Web of Science-from January 1980 to November 2013. Clinical trials and systematic reviews published in English and non-English languages and that evaluated the efficacy and/or cost-effectiveness of interventions of primary prevention for anxiety in primary care in the general adult population were included. Risk of bias was assessed by the Cochrane Risk Bias Tool and Overview Quality Assessment Questionnaire.

Results. No high-quality research was identified that studied primary prevention of anxiety disorders in the adult population in the primary care setting. The few studies that exist focus on the child-adolescent population or on other types of interventions, usually as secondary prevention.

Conclusion. This study emphasizes the need for the development of high-quality clinical trials on the prevention of anxiety disorders in primary care.
\end{abstract}

(c) 2014 Elsevier Inc. All rights reserved.

\section{Introduction}

Anxiety disorders are the most prevalent psychiatric disorders in primary care, with an estimated lifetime prevalence of approximately $16 \%$ in general population (Kessler et al., 2009). All anxiety disorders have been found to be associated with higher disability (Hendriks et al., 2014), and they lead to considerable suffering for the individual, as well as major social, interpersonal and economic costs (Baxter et al., 2014). Probably because of their chronic nature, they also are associated with high comorbidity and usually affect the patient's quality of life and psychosocial functioning (Mendlowicz and Stein, 2000) in addition to a high cost to the health care system and a burden on health resources (Smit et al., 2006).

Their high prevalence and related consequences have led to the development of various prevention programs that have effectively slowed

\footnotetext{
* Corresponding author at: Department of Psychiatry, Miguel Servet University Hospital, 50021 Zaragoza, Spain. Fax: + 34976254006.

E-mail address: jgarcamp@gmail.com (J. García-Campayo).
}

the incidence or minimized the effects of anxiety disorders (e.g., Fisak, Richard, Mann, 2011; Ginsburg, 2009; Kenardy, Mccafferty and Rosa, 2003; van't Veer-Tazelaar et al., 2011; Zalta, 2011).

Prevention of anxiety disorders is necessary to improve the health of the population (Batelaan et al., 2010), and this intervention should be focused on patients with risk factors, with the aim of being as costeffective as possible (Meulenbeek et al., 2008; Smit et al., 2009). The main risk factors identified in previous studies are: to have previously suffered from panic attacks or another anxiety disorder; depression or other affective disorder; and chronic somatic illnesses or stressful life events (such as job loss) (Batelaan et al., 2010; Haro et al., 2006; Moreno-Peral et al., 2014).

The Primary Health Care (PHC) setting is especially ideal for preventing the onset of anxiety disorders because of its accessibility, availability, and continuity of care for the majority of the general population of many countries. Prevention activities are based on the reduction of risk factors and the promotion of protective factors related to anxiety disorders, with the objective of reducing their prevalence and incidence. Evidence-based preventive measures vary in their 
effectiveness depending on their target population, the timing of their implementation, the intervention method used, and the type of anxiety disorder and of risk or protective factors involved (Hosman, Dadds and Raphael, 2005). The aim of our study was to clarify these aspects, with the ultimate objective of determining whether there are disease prevention and health promotion interventions that decrease the incidence and reduce the costs associated with anxiety disorders.

This review is part of a larger project of Spain's Research Network on Prevention Activities and Health Promotion (redIAPP): to develop a complex multi-risk intervention strategy to prevent the most common diseases, like anxiety disorders, and promote healthy aging. The intervention will be constructed from the most effective examples identified in reviews of interventions related to anxiety, depression, alcohol abuse, etc. The target population for this intervention is primary care patients aged 45 to 75 years; therefore, the present review was limited to studies of mature adult populations in the context of primary care.

The objective of our study is to evaluate the efficacy and costeffectiveness of interventions designed for primary prevention of anxiety disorders in the adult population seeking primary care.

\section{Methods}

This review followed PRISMA (Preferred Reporting Items for Systematic Reviews and Meta-Analyses) guidelines (Moher et al., 2009).

\section{Eligibility criteria}

Studies were included if they met the following criteria: a) intervention destined for the general adult population; b) development of a complex, multipleintervention program or strategy of primary prevention in Primary Care; c) comparison with usual care or any intervention; and d) aiming to decrease the incidence of anxiety disorders or to evaluate cost-effectiveness. We included studies published in English and non-English languages.

Initially we looked for systematic reviews and clinical practice guidelines, but because of the low number of studies identified in a preliminary stage it was decided to broaden the search to include randomized controlled trials, meta-analysis, and clinical trials

Data sources and search strategy

A comprehensive literature search was carried out in bibliographical databases from January 1980 to November 2013. The starting date was established because it was when the most modern diagnostic definitions of mental disorders (as DSM) became widely used. The main electronic bibliographic databases selected for this review were PubMed and Embase for health sciences, PsycInfo for psychology, the Cochrane Library for collecting systematic reviews and meta-analysis, and Web of Science with the aim to collect all relevant references. We used controlled vocabulary and free-text terms. The search strategy was developed first in PubMed (Appendix A) and later adapted to all databases. To cover clinical trials from PubMed more effectively, the highly sensitive search filters developed by Haynes et al. were used. The reference lists of important studies on anxiety prevention were reviewed and a manual search on the main primary care journals was conducted, with both strategies producing other studies to analyze.

\section{Selection of studies}

Unduplicated titles and abstracts recovered from databases were screened independently by three review authors. Disagreements between reviewers were resolved by consensus. We excluded the studies that did not meet all of the inclusion criteria (i.e., not the type of study of interest, not carried out in the primary care setting, not a study of the general population, participant ages were outside of the established parameters, the intervention was not appropriate or the results did not refer to lessening the onset of anxiety disorders or evaluate the efficacy or cost-effectiveness of the intervention(s)).

\section{Data extraction and risk of bias}

Three reviewers analyzed independently the information about each study, using a data extraction sheet that was designed for the present review (see
Appendix B). The Cochrane Risk Bias Tool (Higgins, 2008) for clinical trials and Overview Quality Assessment Questionnaire (OQAQ) for systematic reviews (Oxman and Guyatt, 1991) were selected to evaluate risk of bias.

\section{Data analysis}

The main goal was to examine the characteristics of interventions, participants, and outcomes for each study.

Although we intended to estimate the effect size for the various preventive interventions, comparing the incidence of anxiety disorders and the primary measures of study results between the control group and intervention group, because we identified no high-quality studies we were unable to analyze their impact.

\section{Results}

After the initial selection of 957 records, titles and abstracts were screened and 139 references were considered relevant for further analysis (Fig. 1). There were five main reasons for excluding studies: a) no anxiety disorder was included in the aims of the intervention; b) the study was not carried out in primary care; c) target population was different than our population of interest (kids, children, adolescent, older people; healthy professionals, caregivers; etc.); d) the study contained no randomized controlled trials or clinical trials, systematic reviews, meta-analysis; and e) research was not related to an intervention or not to the targeted intervention to prevent the development of anxiety disorders.

After scrutiny of the full texts, none of the 139 references met the inclusion criteria. Our search strategy found no published studies of primary prevention of anxiety disorders in adult populations in the primary care setting.

\section{Discussion}

Despite the proven effectiveness of many anxiety prevention programs, our review of literature identified no study that specifically evaluated the effectiveness of these programs using a clinical trial in the primary care setting. There are various potential explanations for the results obtained. According to the 2004 WHO report on the prevention of mental disorders (Hosman, Dadds, Raphael, 2005), the following are some of the possible interventions for the primary prevention of anxiety:

Public policies on preventing and reducing exposure to traumatic events (child abuse, violence, sexual abuse, traffic accidents, natural disasters)

There is no evidence of the preventive impact of these measures; however, given the nature of these events, a study of preventive programs would not be a clinical trial, but rather a cohort design, with the associated limitations (costs, losses to follow-up). Nonetheless, such prevention policies are very important to the promotion of mental health in our population.

\section{Programs to prevent the development of anxiety disorders}

Most anxiety disorders appear in infancy and adolescence. Therefore, these age groups constitute a fundamental population for primary prevention. Most of the clinical trials on the prevention of anxiety have involved children and adolescents, and the main setting for interventions is the school, not primary care. Many existing programs have demonstrated their effectiveness in preventing symptoms of anxiety and the onset of the disorder (Mychailyszyn et al., 2012). One of the most effective interventions is the FRIENDS program (Dadds et al., 1997; Lowry-Webster, Barrett, and Dadds;, 2001; Lowry-Webster, Barrett and Lock;, 2003), a cognitive behavior program designed for children aged 6 to 16 years that is carried out in schools as well as health care centers and hospitals. This program improves symptoms of anxiety 

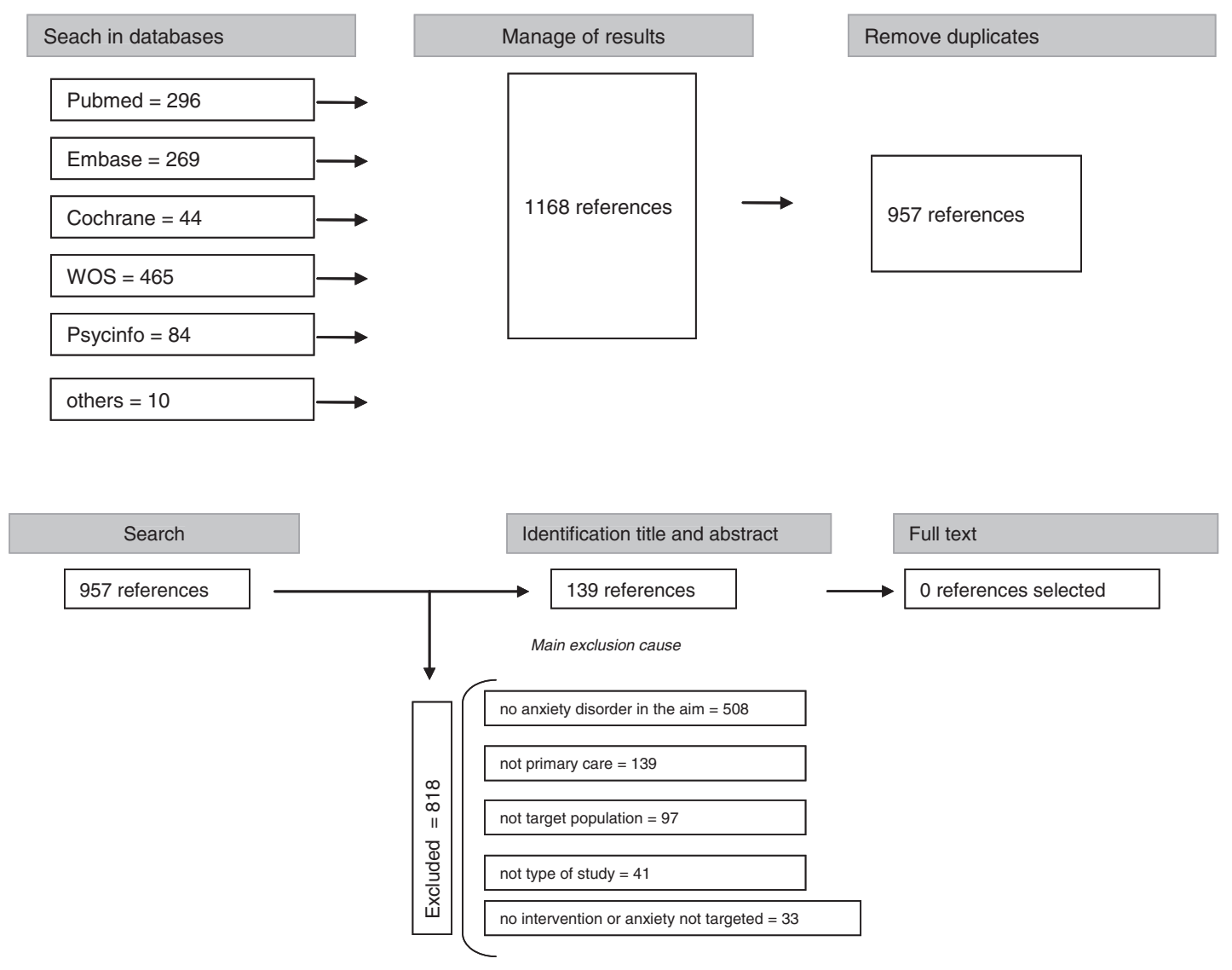

Fig. 1. Study flow diagram.

and depression, with especially noteworthy results among children with high anxiety levels, and the effects persist over time. Other trials of note developed interventions focused primarily on the prevention of depression, although they also prevented or improved symptoms of anxiety (Roberts et al., 2003; Seligman, Schulman, DeRubeis, Hollon, 1999; Seligman, Schulman and Tryon, 2007). Interventions designed for the primary prevention of anxiety are effective, but their natural setting is the school. It could be interesting to study the most effective preventive interventions and transfer them to the primary care setting. In our study, this objective does not apply because the intervention that would be designed would be directed exclusively to the adult population.

On the other hand, some studies have reduced anxiety in patients with various pathologies such as those analyzed in Herring, O'Connor, Dishman (2010). These studies have shown that physical exercise significantly reduces symptoms of anxiety in chronic diseases (multiple sclerosis, psychological disorders, cancer, chronic obstructive pulmonary disorder, chronic pain, and others).

Interventions after a trauma: debriefing and early treatments to prevent post-traumatic stress disorder

Debriefing does not prevent post-traumatic stress or other psychiatric disorders. There is also no evidence that it reduces anxiety (van Emmerik et al., 2002); in fact, it might increase the long-term risk of post-traumatic stress disorder (Rose et al., 2002). Other types of early treatment after a trauma have been shown to be effective in preventing this disorder (Bryant et al., 1998). Nonetheless, very few controlled randomized trials have been done that included therapeutic interventions directed at the initial trauma with the goal of preventing posttraumatic stress; the few that exist are of varying quality and included small samples, probably due to ethical considerations, among other reasons (e.g., limited resources, technical difficulties and other barriers to clinical trial implementation (Marshall and Suh, 2003).

A feasibility study by Batelaan et al. (2012), which had the objective of researching the willingness to participate in screening and interventions, reveals other possible reasons for the limited studies found. They conclude that the feasibility of preventing anxiety disorders in primary care is very poor, as only a minority of the target population was willing to be screened (17.3\%), even when patients knew that they are at risk.

Perhaps the main problem encountered by the present study was the limitations imposed by the criteria chosen for inclusion and exclusion of published clinical trials. In addition to the scarcity of studies on the prevention of anxiety disorders, we limited the selection of articles to studies of high quality, with interventions carried out in primary care and focused on adults in a specific age range (published studies have included children, adolescents, and the elderly population) of the general population (not health professionals or diagnosed patients). Under these criteria, no studies met the requirements and so there were none to be analyzed.

\section{Study implications}

The finding that there have been no clinical trials of the primary prevention of anxiety disorders indicates a gap in the literature. This should encourage researchers and primary care health professionals to develop programs based on existing studies that have demonstrated their effectiveness in prevention of these disorders in populations of children and adolescents. In the primary care setting, such programs could reduce both the social and economic costs of these disorders.

Nonetheless, other types of studies, such as qualitative research, are needed to evaluate the reasons and obstacles that have kept these types of interventions from being carried out and described in the literature. This would clarify, as much as possible, the factors that limit their 
development; however, some of the reasons-such as those linked to the workload of primary care professionals-will be very difficult to overcome.

\section{Conclusions}

Despite the evidence of the effectiveness of primary prevention programs in reducing the incidence of anxiety, almost no studies have been carried out in primary care settings. The few studies identified in this context were directed at populations of children and adolescents or focused on other types of intervention, usually secondary prevention. A few studies were identified that analyzed interventions after a trauma experience, which were designed to prevent the onset of post-traumatic stress disorder. However, these were not randomized clinical trials, mainly because of ethical considerations.

This review of literature highlights the need to develop high-quality programs and clinical trials focused on the prevention of anxiety disorders in primary care settings.

\section{Funding}

The project has received funding from a Network for Prevention and Health Promotion in Primary Care (redIAPP, RD12/0005) grant and a research project grant (PI12/01914) from the Instituto de Salud Carlos III (Institute of Health Carlos III) of the Ministry of Economy and Competitiveness (Spain), co-financed with European Union ERDF funds.

\section{Conflict of interest statement}

No authors have any competing interests.

\section{Contributions of authors}

JGC and YL developed the protocol and selection criteria. MS was involved in designing and carrying out the search strategy. JGC, YL, and EA were involved in applying the first round of article selection. MCP, MP, and RM extracted the final records. All authors participated in drafting the manuscript and approved the final version submitted for publication.

\section{Appendix A. Supplementary data}

Supplementary data to this article can be found online at http://dx. doi.org/10.1016/j.ypmed.2014.10.015.

\section{References}

Batelaan, N.M., Smit, F., De Graaf, R., Van Balkom, A.J.L.M., Vollebergh, W.A.M., Beekman A.T.F., 2010. Identifying target groups for the prevention of anxiety disorders in the general population. Acta Psychiatr. Scand. 122 (1), 56-65.

Batelaan, N.M., Smit, J.H., Cuijpers, P., van Marwijk, H.W., Terluin, B., van Balkom, A.J. 2012. Prevention of anxiety disorders in primary care: a feasibility study. BMC Psychiatry 12 (1), 206

Baxter, A.J., Vos, T., Scott, K.M., Ferrari, A.J., Whiteford, H.A., 2014. The global burden of anxiety disorders in 2010. Psychol. Med. 1-12. http://dx.doi.org/10.1017/S0033291713003243.

Bryant, R.A., Harvey, A.G., Dang, S.T., Sackville, T., Basten, C., 1998. Treatment of acute stress disorder: a comparison of cognitive-behavioral therapy and supportive counseling. J. Consult. Clin. Psychol. 66 (5), 862.

Dadds, M.R., Spence, S.H., Holland, D.E., Barrett, P.M., Laurens, K.R., 1997. Prevention and early intervention for anxiety disorders: a controlled trial. J. Consult. Clin. Psychol. 65 (4), 627.
Fisak Jr., B.J., Richard, D., Mann, A., 2011. The prevention of child and adolescent anxiety: a meta-analytic review. Prev. Sci. 12 (3), 255-268. http://dx.doi.org/10.1007/s11121011-0210-0.

Ginsburg, G.S., 2009. The child anxiety prevention study: intervention model and primary outcomes. J. Consult. Clin. Psychol. 77 (3), 580. http://dx.doi.org/10.1037/ a0014486.

Haro, J.M., Palacín, C., Vilagut, G., et al., 2006. Prevalencia de los trastornos mentales y factores asociados: resultados del estudio ESEMeD-España. Med. Clin. 126 (12), 445-451.

Haynes, et al., d. Summary of Enhancements for Clinical and Health Services Research Queries for PubMed for StudiesRetrieved from http://www.nlm.nih.gov/pubs/ techbull/jf04/cq info.html.

Hendriks, S.M., Spijker, J., Licht, C.M., et al., 2014. Disability in anxiety disorders. J. Affect. Disord. 166, 227-233.

Herring, M.P., O'Connor, P.J., Dishman, R.K., 2010. The effect of exercise training on anxiety symptoms among patients: a systematic review. Arch. Intern. Med. 170 (4), 321-331. http://dx.doi.org/10.1001/archinternmed.2009.530.

Higgins, J.P. (Ed.), 2008. Cochrane Handbook for Systematic Reviews of Interventions vol. 5. Wiley-Blackwell, Chichester.

Hosman, C., Dadds, M., Raphael, B., 2005. Prevention of anxiety disorders. In: Hosman, C., Jané-Llopis, E., Saxena, S. (Eds.), Prevention of Mental Disorders: Effective Interventions and Policy Options. Oxford University Press, Oxford.

Kenardy, J., McCafferty, K., Rosa, V., 2003. Internet-delivered indicated prevention for anxiety disorders: a randomized controlled trial. Behav. Cogn. Psychother. 31 (03), 279-289.

Kessler, R., Aguilar-Gaxiola, S., Alonso, J., et al., 2009. Special articles. The global burden of mental disorders: an update from the WHO World Mental Health (WMH) surveys. Epidemiol. Psichiatr. Soc. 18 (1), 23.

Lowry-Webster, H.M., Barrett, P.M., Dadds, M.R., 2001. A universal prevention trial of anxiety and depressive symptomatology in childhood: preliminary data from an Australian study. Behav. Chang. 18 (01), 36-50.

Lowry-Webster, H.M., Barrett, P.M., Lock, S., 2003. A universal prevention trial of anxiety symptomology during childhood: results at 1-year follow-up. Behav. Chang. 20 (01), 25-43.

Marshall, R.D., Suh, E.J., 2003. Contextualizing trauma: using evidence-based treatments in a multicultural community after 9/11. Psychiatry Q. 74 (4), 401-420.

Mendlowicz, M.V., Stein, M.B., 2000. Quality of life in individuals with anxiety disorders. Am. J. Psychiatr. 157 (5), 669-682.

Meulenbeek, P., Willemse, G., Smit, F., van Balkom, A., Spinhoven, P., Cuijpers, P., 2008. Early intervention in panic: randomized controlled trial and cost-effectiveness analysis. Trials 9 (1), 67.

Moher, D., Liberati, A., Tetzlaff, J., Altman, D.G., 2009. Preferred reporting items for systematic reviews and meta-analyses: the PRISMA statement. Ann. Intern. Med. 151 (4), 264-269.

Moreno-Peral, P., Conejo-Cerón, S., Motrico, E., et al., 2014. Risk factors for the onset of panic and generalised anxiety disorders in the general adult population. A systematic review of cohort studies. J. Affect. Disord. 168, 337-348.

Mychailyszyn, M.P., Brodman, D.M., Read, K.L., Kendall, P.C., 2012. Cognitive-behavioral school-based interventions for anxious and depressed youth: a meta-analysis of outcomes. Clin. Psychol. Sci. Pract. 19 (2), 129-153.

Oxman, A.D., Guyatt, G.H., 1991. Validation of an index of the quality of review articles. J. Clin. Epidemiol. 44 (11), 1271-1278.

Roberts, C., Kane, R., Thomson, H., Bishop, B., Hart, B., 2003. The prevention of depressive symptoms in rural school children: a randomized controlled trial. J. Consult. Clin. Psychol. 71 (3), 622

Rose, S., Bisson, J., Churchill, R., Wessely, S., 2002. Psychological debriefing for preventing post traumatic stress disorder (PTSD). Cochrane Database Syst. Rev. 2 (2)

Seligman, M.E., Schulman, P., DeRubeis, R.J., Hollon, S.D., 1999. The prevention of depression and anxiety. Prev. Treat. 2 (1), 8a.

Seligman, M.E., Schulman, P., Tryon, A.M., 2007. Group prevention of depression and anxiety symptoms. Behav. Res. Ther. 45 (6), 1111-1126.

Smit, F., Cuijpers, P., Oostenbrink, J., Batelaan, N., de Graaf, R., Beekman, A., 2006. Costs of nine common mental disorders: implications for curative and preventive psychiatry. J. Ment. Health Policy Econ. 9, 193-200.

Smit, F., Willemse, G., Meulenbeek, P., et al., 2009. Cost effectiveness and resource allocation. Cost Eff. Res. Allocation 7, 8.

Van Emmerik, A.A., Kamphuis, J.H., Hulsbosch, A.M., Emmelkamp, P.M., 2002. Single session debriefing after psychological trauma: a meta-analysis. Lancet 360 (9335), 766-771.

van't Veer-Tazelaar, P.J., van Marwijk, H.W., van Oppen, P., et al., 2011. Prevention of latelife anxiety and depression has sustained effects over 24 months: a pragmatic randomized trial. Am. J. Geriatr. Psychiatry 19 (3), 230-239.

Zalta, A.K., 2011. A meta-analysis of anxiety symptom prevention with cognitivebehavioral interventions. J. Anxiety Disord. 25 (5), 749-760. 